\title{
Counting discriminants of number fields
}

\author{
par Henri COHEN, Francisco DiAZ Y DiAZ et Michel OliVieR
}

Dedicated to Michael Pohst for his 60th birthday

\begin{abstract}
RÉSumÉ. Pour tout groupe de permutations transitif sur $n$ lettres $G$ avec $n \leq 4$ nous donnons sans démonstration des résultats, des conjectures et des calculs numériques sur le nombre de discriminants de corps de nombres $L$ de degré $n$ sur $\mathbb{Q}$ tels que le groupe de Galois de la clôture galoisienne de $L$ soit isomorphe à $G$.
\end{abstract}

Abstract. For each transitive permutation group $G$ on $n$ letters with $n \leq 4$, we give without proof results, conjectures, and numerical computations on discriminants of number fields $L$ of degree $n$ over $\mathbb{Q}$ such that the Galois group of the Galois closure of $L$ is isomorphic to $G$.

\section{Introduction}

The aim of this paper is to regroup results and conjectures on discriminant counts of number fields of degree less than or equal to 4 , from a theoretical, practical, and numerical point of view. Proofs are given elsewhere, see the bibliography. We only consider absolute number fields.

If $G$ is a permutation group on $n$ letters, we write

$$
\Phi_{n}(G, s)=\sum_{L / \mathbb{Q}} \frac{1}{|d(L)|^{s}} \text { and } N_{n}(G, X)=\sum_{L / \mathbb{Q},|d(L)| \leq X} 1,
$$

where in both cases the summation is over isomorphism classes of number fields $L$ of degree $n$ over $\mathbb{Q}$ such that the Galois group of the Galois closure of $L$ is isomorphic to $G$ and $d(L)$ denotes the absolute discriminant of $L$. When we specify the signature $\left(r_{1}, r_{2}\right)$, we will instead write $\Phi_{r_{1}, r_{2}}(G, s)$ and $N_{r_{1}, r_{2}}(G, X)$.

We denote by $C_{n}$ the cyclic group of order $n$, by $S_{n}$ the symmetric group on $n$ letters, by $A_{n}$ the alternating group on $n$ letters, and by $D_{n}$ the dihedral group with $2 n$ elements.

We note that certain authors, in particular Datskovsky, Wright and Yukie (see [20], [32], [34]) count number fields in a fixed algebraic closure of $\mathbb{Q}$. This is the same as $N_{n}(G, X)$ when $G$ is of cardinality equal to $n$, i.e., when

Manuscrit reçu le 19 octobre 2005. 
the extensions $L$ are Galois. Otherwise, in the range of our study $(n \leq 4)$, their count is equal to $m(G) N_{n}(G, X)$ with $m\left(S_{3}\right)=3, m\left(D_{4}\right)=2$, and $m\left(A_{4}\right)=m\left(S_{4}\right)=4$.

For each group $G$, we give the results in the following form. Whenever possible, we first give expressions for $\Phi_{n}(G, s)$ and $\Phi_{r_{1}, r_{2}}(G, s)$ which are as explicit as possible. Then we give asymptotic formulas for $N_{n}(G, X)$ and $N_{r_{1}, r_{2}}(G, X)$ which are usually directly deduced from the formula for $\Phi_{n}(G, s)$ and $\Phi_{r_{1}, r_{2}}(G, s)$, in the form $N_{n}(G, X)=P_{n}(G, X)+R_{n}(G, X)$ and $N_{r_{1}, r_{2}}(G, X)=P_{r_{1}, r_{2}}(G, X)+R_{r_{1}, r_{2}}(G, X)$, where the $P(G, X)$ are main terms, and the quantities $R(G, X)$ (which denote any one of $R_{n}(G, X)$ or $\left.R_{r_{1}, r_{2}}(G, X)\right)$ are error terms. We then give conjectural estimates of the form $R(G, X)=\widetilde{O}\left(X^{\alpha}\right)$ for some exponent $\alpha$, where we use the convenient "soft O" notation: $f(X)=\widetilde{O}\left(X^{\alpha}\right)$ means that $f(X)=O\left(X^{\alpha+\varepsilon}\right)$ for any $\varepsilon>0$. (note that this does not necessarily mean $f(X)=O\left(X^{\alpha} \log (X)^{\beta}\right)$ for some $\beta$ ). In most cases, a suitable value for $\alpha$ can be rigorously obtained by complex integration methods, but we have not made any attempt in this direction, citing existing references when possible.

Note that those among the explicit constants occurring in the main terms which occur as products or sums over primes are all given numerically to at least 30 decimal digits. This is computed using a now rather standard method which can be found for example in [10].

Finally, we give tables of $N_{r_{1}, r_{2}}\left(G, 10^{k}\right)$ for all possible signatures $\left(r_{1}, r_{2}\right)$ and increasing values of $k$, as well as a comment on the comparison between this data with the most refined result or conjecture on the asymptotic behavior. To save space, we do not give $N\left(G, 10^{k}\right)$ which is of course trivially obtained by summing over all possible signatures. The upper bound chosen for $k$ depends on the time and space necessary to compute the data: usually a few weeks of CPU time and 1GB of RAM.

We have noticed that in most of the tables that we give, the error term (which we do not indicate explicitly) changes sign and is rather small, indicating both that there is no systematic bias, in other words no additional main term, and that the conjectured exponent in the error term is close to the correct value. Whenever there seems to be such a systematic bias, a least squares method has been used to find a conjectured additional main term, and these terms have been used in the tables. When appropriate, this is indicated in the corresponding sections.

It should be stressed that although we only give the number of suitable fields, the same methods can also be used to compute explicitly a defining equation for these number fields, but the storage problem makes this impractical for more than a few million fields. See [14] and [15] for details. 
General references. Outside from specific references which will be given in each section, the following papers give general results and/or conjectures. The paper of Wright [32] gives a general formula for $N_{n}(G, X)$ for abelian groups $G$ (and even for general abelian extensions of number fields). The exponents of $X$ and $\log X$ are easily computable, however the multiplicative constant is only given as an adelic integral which is in principle computable, but in practice very difficult to compute. In fact, for general base fields these constants have been computed only in very few cases, and by quite different methods, in particular by the authors (see [16], [17], and [18]).

The thesis and paper of Mäki [25] and [26] give $\Phi_{n}(G, X)$ and estimates for $N_{n}(G, X)$ with error terms (easily deduced from $\Phi_{n}(G, X)$ by contour integration) again in the case of abelian groups $G$, but only for absolute extensions, i.e., when the base field is $\mathbb{Q}$, as we do here. This nicely complements the results of Wright, but is limited to the base field $\mathbb{Q}$. She does not give results with signatures, although they could probably be obtained using her methods.

The papers of Malle [27] and [28] give very general and quite precise conjectures on $N_{n}(G, X)$ for arbitrary transitive subgroups $G$ of $S_{n}$, up to an unknown multiplicative constant, as well as results and heuristics supporting these conjectures. Although the conjectures must be corrected as stated (see [24] for a counter-example), the general form is believed to be correct.

Finally, the ICM talk [12] can be considered as a summary of the present paper.

\section{Degree 2 fields with $\mathrm{G} \simeq \mathrm{C}_{2}$}

2.1. Dirichlet series and asymptotic formulas. The results are elementary.

Dirichlet series:

$$
\begin{aligned}
\Phi_{2}\left(C_{2}, s\right) & =\left(1+\frac{1}{2^{2 s}}+\frac{2}{2^{3 s}}\right) \prod_{p \equiv 1(\bmod 2)}\left(1+\frac{1}{p^{s}}\right)-1 \\
& =\left(1-\frac{1}{2^{s}}+\frac{2}{2^{2 s}}\right) \prod_{p}\left(1+\frac{1}{p^{s}}\right)-1 \\
& =\left(1-\frac{1}{2^{s}}+\frac{2}{2^{2 s}}\right) \frac{\zeta(s)}{\zeta(2 s)}-1 \\
\Phi_{2,0}\left(C_{2}, s\right) & =\frac{1}{2} \Phi_{2}\left(C_{2}, s\right)+\frac{1}{2}\left(1-\frac{1}{2^{2 s}}\right) \prod_{p \equiv 1(\bmod 2)}\left(1+\frac{(-1)^{(p-1) / 2}}{p^{s}}\right)-\frac{1}{2} \\
\Phi_{0,1}\left(C_{2}, s\right) & =\Phi_{2}\left(C_{2}, s\right)-\Phi_{2,0}\left(C_{2}, s\right) .
\end{aligned}
$$


Asymptotic formulas:

$$
\begin{aligned}
N_{2}\left(C_{2}, X\right) & =c\left(C_{2}\right) X+R_{2}\left(C_{2}, X\right) \\
N_{2,0}\left(C_{2}, X\right) & =\frac{c\left(C_{2}\right)}{2} X+R_{2,0}\left(C_{2}, X\right) \\
N_{0,1}\left(C_{2}, X\right) & =\frac{c\left(C_{2}\right)}{2} X+R_{0,1}\left(C_{2}, X\right),
\end{aligned}
$$

with

$$
c\left(C_{2}\right)=\frac{1}{\zeta(2)}=\frac{6}{\pi^{2}}
$$

and

$$
R\left(C_{2}, X\right)=O\left(X^{1 / 2} \exp \left(-c(\log X)^{3 / 5}(\log \log X)^{-1 / 5}\right)\right)
$$

for some positive constant $c$, and under the Riemann Hypothesis

$$
R\left(C_{2}, X\right)=\widetilde{O}\left(X^{8 / 25}\right)
$$

(see for example [31], Notes du Chapitre I.3). It is conjectured, and this is strongly confirmed by the data, that $R\left(C_{2}, X\right)=\widetilde{O}\left(X^{1 / 4}\right)$, hence to compare with the data we use $\alpha=1 / 4$.

2.2. Tables. These tables have been computed using the methods explained in [8].

\begin{tabular}{|l||l|l|}
\hline$X$ & $N_{2,0}\left(C_{2}, X\right)$ & $N_{0,1}\left(C_{2}, X\right)$ \\
\hline \hline $10^{1}$ & 2 & 4 \\
$10^{2}$ & 30 & 31 \\
$10^{3}$ & 302 & 305 \\
$10^{4}$ & 3043 & 3043 \\
$10^{5}$ & 30394 & 30392 \\
$10^{6}$ & 303957 & 303968 \\
$10^{7}$ & 3039653 & 3039632 \\
$10^{8}$ & 30396324 & 30396385 \\
$10^{9}$ & 303963559 & 303963510 \\
$10^{10}$ & 3039635379 & 3039635443 \\
$10^{11}$ & 30396355148 & 30396355052 \\
$10^{12}$ & 303963551039 & 303963550712 \\
$10^{13}$ & 3039635509103 & 3039635509360 \\
$10^{14}$ & 30396355093462 & 30396355092880 \\
$10^{15}$ & 303963550926173 & 303963550926479 \\
\hline
\end{tabular}




\begin{tabular}{|l||l|l|}
\hline$X$ & $N_{2,0}\left(C_{2}, X\right)$ & $N_{0,1}\left(C_{2}, X\right)$ \\
\hline \hline $10^{16}$ & 3039635509271389 & 3039635509273025 \\
$10^{17}$ & 30396355092697223 & 30396355092696593 \\
$10^{18}$ & 303963550927008744 & 303963550927017751 \\
$10^{19}$ & 3039635509270131961 & 3039635509270110507 \\
$10^{20}$ & 30396355092701313737 & 30396355092701291066 \\
$10^{21}$ & 303963550927013401272 & 303963550927013312649 \\
$10^{22}$ & 3039635509270133130535 & 3039635509270133092175 \\
$10^{23}$ & 30396355092701331456323 & 30396355092701331531457 \\
$10^{24}$ & 303963550927013314010676 & 303963550927013314554179 \\
$10^{25}$ & 3039635509270133143448215 & 3039635509270133143069580 \\
\hline
\end{tabular}

The relative error between the actual data and the predictions varies between $-0.57 \%$ and $0.57 \%$.

\section{Degree 3 fields with $\mathrm{G} \simeq \mathrm{C}_{3}$}

3.1. Dirichlet series and asymptotic formulas. The results are due to Cohn [19], and can easily be obtained from the much older characterization of cyclic cubic fields due to Hasse [23], see for example [6], Section 6.4.2.

Dirichlet series:

$$
\begin{aligned}
\Phi_{3}\left(C_{3}, s\right) & =\frac{1}{2}\left(1+\frac{2}{3^{4 s}}\right) \prod_{p \equiv 1}\left(1+\frac{2}{p^{2 s}}\right)-\frac{1}{2} \\
\Phi_{3,0}\left(C_{3}, s\right) & =\Phi_{3}\left(C_{3}, s\right) \\
\Phi_{1,1}\left(C_{3}, s\right) & =0 .
\end{aligned}
$$

Asymptotic formulas:

$$
\begin{aligned}
N_{3}\left(C_{3}, X\right) & =c\left(C_{3}\right) X^{1 / 2}+R_{3}\left(C_{3}, X\right) \\
N_{3,0}\left(C_{3}, X\right) & =N_{3}\left(C_{3}, X\right) \\
N_{1,1}\left(C_{3}, X\right) & =0
\end{aligned}
$$

with

$$
\begin{aligned}
c\left(C_{3}\right) & =\frac{11 \sqrt{3}}{36 \pi} \prod_{p \equiv 1}\left(1-\frac{2}{p(p+1)}\right) \\
& =0.1585282583961420602835078203575 \ldots
\end{aligned}
$$

and $R_{3}\left(C_{3}, X\right)=\widetilde{O}\left(X^{1 / 3}\right)$.

It is reasonable to conjecture that we should have $R_{3}\left(C_{3}, X\right)=\widetilde{O}\left(X^{1 / 6}\right)$, hence to compare with the numerical data we use $\alpha=1 / 6$. 
3.2. Table. This table has been computed using the methods explained in $[8]$.

\begin{tabular}{|l||l||l||l||l||l|}
\hline$X$ & $N_{3}\left(C_{3}, X\right)$ & $X$ & $N_{3}\left(C_{3}, X\right)$ & $X$ & $N_{3}\left(C_{3}, X\right)$ \\
\hline \hline $10^{1}$ & 0 & $10^{14}$ & 1585249 & $10^{27}$ & 5013103697105 \\
$10^{2}$ & 2 & $10^{15}$ & 5013206 & $10^{28}$ & 15852825840369 \\
$10^{3}$ & 5 & $10^{16}$ & 15852618 & $10^{29}$ & 50131036986701 \\
$10^{4}$ & 16 & $10^{17}$ & 50131008 & $10^{30}$ & 158528258396671 \\
$10^{5}$ & 51 & $10^{18}$ & 158528150 & $10^{31}$ & 501310370020343 \\
$10^{6}$ & 159 & $10^{19}$ & 501309943 & $10^{32}$ & 1585282583932681 \\
$10^{7}$ & 501 & $10^{20}$ & 1585282684 & $10^{33}$ & 5013103700345884 \\
$10^{8}$ & 1592 & $10^{21}$ & 5013103291 & $10^{34}$ & 15852825839615504 \\
$10^{9}$ & 5008 & $10^{22}$ & 15852826251 & $10^{35}$ & 50131037003076114 \\
$10^{10}$ & 15851 & $10^{23}$ & 50131036382 & $10^{36}$ & 158528258396205064 \\
$10^{11}$ & 50152 & $10^{24}$ & 158528255967 & $10^{37}$ & 501310370031289126 \\
$10^{12}$ & 158542 & $10^{25}$ & 501310368157 & & \\
$10^{13}$ & 501306 & $10^{26}$ & 1585282578080 & & \\
\hline
\end{tabular}

The relative error between the actual data and the predictions varies between $-0.68 \%$ and $0.33 \%$.

\section{Degree 3 fields with $G \simeq S_{3} \simeq D_{3}$}

4.1. Dirichlet series and asymptotic formulas. The main terms in the asymptotic formulas are due to Davenport and Heilbronn [21], [22]. The other terms are conjectural and can be attributed to Datskovsky-Wright [20] and Roberts [30].

Dirichlet series:

In this case, the Dirichlet series do not seem to have any nice form.

Asymptotic formulas:

$$
\begin{aligned}
N_{3}\left(S_{3}, X\right) & =c\left(S_{3}\right) X+c^{\prime}\left(S_{3}\right) X^{5 / 6}-\frac{c\left(C_{3}\right)}{3} X^{1 / 2}+R_{3}\left(S_{3}, X\right) \\
N_{3,0}\left(S_{3}, X\right) & =\frac{c\left(S_{3}\right)}{4} X+\frac{c^{\prime}\left(S_{3}\right)}{\sqrt{3}+1} X^{5 / 6}-\frac{c\left(C_{3}\right)}{3} X^{1 / 2}+R_{3,0}\left(S_{3}, X\right) \\
N_{1,1}\left(S_{3}, X\right) & =\frac{3}{4} c\left(S_{3}\right) X+\frac{\sqrt{3}}{\sqrt{3}+1} c^{\prime}\left(S_{3}\right) X^{5 / 6}+R_{1,1}\left(S_{3}, X\right),
\end{aligned}
$$


with

$$
\begin{aligned}
c\left(S_{3}\right) & =\frac{1}{3 \zeta(3)}=0.27730245752690248956104209294 \ldots \\
c^{\prime}\left(S_{3}\right) & =\frac{4(\sqrt{3}+1)}{5 \Gamma(2 / 3)^{3}} \frac{\zeta(1 / 3)}{\zeta(5 / 3)} \\
& =-0.40348363666394679863364025671534 \ldots
\end{aligned}
$$

and

$$
R\left(S_{3}, X\right)=\widetilde{O}\left(X^{19 / 20}\right) .
$$

This remainder term is due to Belabas-Bhargava-Pomerance [4], and evidently, in these estimates the remainder term is of larger order than the additional main term. The reason that we have given this additional term is that much more is conjectured to be true. From heuristics of Roberts and Wright (see [33] and [30]), it is believed that $R\left(S_{3}, X\right)$ is negligible compared to the additional main term, in other words that $R\left(S_{3}, X\right)=o\left(X^{1 / 2}\right)$. Thus, to compare with the numerical data we use these additional main terms and choose $\alpha=1 / 2$, although the tables would seem to indicate that even $\alpha=5 / 12$ could be possible.

4.2. Tables. These tables have been computed by Belabas in [2] using his methods, based on the Davenport-Heilbronn theory, and also explained in detail in [7], Chapter 8. It should not be too difficult to extend them to $X=10^{13}$, say, using the improved methods given in [3].

\begin{tabular}{|l||l|l|}
\hline$X$ & $N_{3,0}\left(S_{3}, X\right)$ & $N_{1,1}\left(S_{3}, X\right)$ \\
\hline \hline $10^{1}$ & 0 & 0 \\
$10^{2}$ & 0 & 7 \\
$10^{3}$ & 22 & 127 \\
$10^{4}$ & 366 & 1520 \\
$10^{5}$ & 4753 & 17041 \\
$10^{6}$ & 54441 & 182417 \\
$10^{7}$ & 592421 & 1905514 \\
$10^{8}$ & 6246698 & 19609185 \\
$10^{9}$ & 64654353 & 199884780 \\
$10^{10}$ & 661432230 & 2024660098 \\
$10^{11}$ & 6715773873 & 20422230540 \\
\hline
\end{tabular}

The relative error between the actual data and the predictions varies between $-0.2 \%$ and $0.04 \%$. 


\section{Degree 4 fields with $\mathrm{G} \simeq \mathrm{C}_{4}$}

5.1. Dirichlet series and asymptotic formulas. The results are not difficult. The paper which is cited in this context is [1], which unfortunately contains several misprints. These are corrected in the papers of Mäki [25] and ours, in particular here.

Dirichlet series:

$$
\begin{aligned}
\Phi_{4}\left(C_{4}, s\right)= & \frac{\zeta(2 s)}{2 \zeta(4 s)}\left(\left(1-\frac{1}{2^{2 s}}+\frac{2}{2^{4 s}}+\frac{4}{2^{11 s}+2^{9 s}}\right) \prod_{\substack{p \equiv 1 \\
(\bmod 4)}}\left(1+\frac{2}{p^{3 s}+p^{s}}\right)\right. \\
& \left.-\left(1-\frac{1}{2^{2 s}}+\frac{2}{2^{4 s}}\right)\right) \\
\Phi_{4,0}\left(C_{4}, s\right)= & \frac{1}{2} \Phi_{4}\left(C_{4}, s\right)+\frac{L\left(2 s,\left(\frac{-4}{\cdot}\right)\right)}{4 \zeta(4 s)}\left(\prod_{\substack{p \equiv 1 \\
(\bmod 4)}}\left(1+\frac{2(-1)^{(p-1) / 4}}{p^{3 s}+p^{s}}\right)-1\right) \\
\Phi_{2,1}\left(C_{4}, s\right)= & 0 \\
\Phi_{0,2}\left(C_{4}, s\right)= & \Phi_{4}\left(C_{4}, s\right)-\Phi_{4,0}\left(C_{4}, s\right) .
\end{aligned}
$$

Asymptotic formulas:

$$
\begin{aligned}
N_{4}\left(C_{4}, X\right) & =c\left(C_{4}\right) X^{1 / 2}+c^{\prime}\left(C_{4}\right) X^{1 / 3}+R_{4}\left(C_{4}, X\right) \\
N_{4,0}\left(C_{4}, X\right) & =\frac{c\left(C_{4}\right)}{2} X^{1 / 2}+\frac{c^{\prime}\left(C_{4}\right)}{2} X^{1 / 3}+R_{4,0}\left(C_{4}, X\right) \\
N_{2,1}\left(C_{4}, X\right) & =0 \\
N_{0,2}\left(C_{4}, X\right) & =\frac{c\left(C_{4}\right)}{2} X^{1 / 2}+\frac{c^{\prime}\left(C_{4}\right)}{2} X^{1 / 3}+R_{0,2}\left(C_{4}, X\right),
\end{aligned}
$$

with

$$
\begin{aligned}
c\left(C_{4}\right) & =\frac{3}{\pi^{2}}\left(\left(1+\frac{\sqrt{2}}{24}\right) \prod_{p \equiv 1(\bmod 4)}\left(1+\frac{2}{p^{3 / 2}+p^{1 / 2}}\right)-1\right) \\
& =0.1220526732513967609226080528965 \ldots \\
c^{\prime}\left(C_{4}\right) & =\frac{3+2^{-1 / 3}+2^{-2 / 3}}{1+2^{-2 / 3}} \frac{\zeta(2 / 3)}{4 \pi \zeta(4 / 3)} \prod_{p \equiv 1(\bmod 4)}\left(1+\frac{2}{p+p^{1 / 3}}\right)\left(\frac{1-1 / p}{1+1 / p}\right) \\
& =-0.11567519939427878830185483678 \ldots
\end{aligned}
$$

Although easy to obtain by contour integration, we have not found the additional $X^{1 / 3}$ main term in the literature.

It is reasonable to conjecture that we should have $R\left(C_{4}, X\right)=\widetilde{O}\left(X^{1 / 6}\right)$, hence to compare with the numerical data we use $\alpha=1 / 6$, although the tables seem to indicate that even $\alpha=1 / 8$ could be possible. 
5.2. Tables. These tables have been computed using the methods explained in [8].

\begin{tabular}{|l||l|l|}
\hline$X$ & $N_{4,0}\left(C_{4}, X\right)$ & $N_{0,2}\left(C_{4}, X\right)$ \\
\hline \hline $10^{1}$ & 0 & 0 \\
$10^{2}$ & 0 & 0 \\
$10^{3}$ & 0 & 1 \\
$10^{4}$ & 6 & 4 \\
$10^{5}$ & 15 & 17 \\
$10^{6}$ & 59 & 54 \\
$10^{7}$ & 182 & 181 \\
$10^{8}$ & 586 & 582 \\
$10^{9}$ & 1867 & 1865 \\
$10^{10}$ & 5966 & 5964 \\
$10^{11}$ & 19017 & 19028 \\
$10^{12}$ & 60456 & 60469 \\
$10^{13}$ & 191736 & 191764 \\
$10^{14}$ & 607589 & 607609 \\
$10^{15}$ & 1924160 & 1924059 \\
$10^{16}$ & 6090130 & 6090110 \\
$10^{17}$ & 19271385 & 19271321 \\
$10^{18}$ & 60968525 & 60968399 \\
$10^{19}$ & 192857593 & 192857870 \\
$10^{20}$ & 609994937 & 609994964 \\
$10^{21}$ & 1929244391 & 1929243674 \\
$10^{22}$ & 6101387381 & 6101387860 \\
$10^{23}$ & 19295537531 & 19295537010 \\
$10^{24}$ & 61020552533 & 61020552938 \\
$10^{25}$ & 192969762398 & 192969758223 \\
$10^{26}$ & 610236520653 & 610236519548 \\
$10^{27}$ & 1929764373961 & 1929764373161 \\
$10^{28}$ & 6102509058257 & 6102509054460 \\
$10^{29}$ & 19297953643936 & 19297953644691 \\
$10^{30}$ & 61025758244048 & 61025758248309 \\
$10^{31}$ & 192980974911603 & 192980974923193 \\
$10^{32}$ & 610260681684841 & 610260681669563 \\
\hline
\end{tabular}

The relative error between the actual data and the predictions varies between $-0.64 \%$ and $0.45 \%$, and seems clearly to tend to 0 as $k \rightarrow \infty$. 


\section{Degree 4 fields with $\mathrm{G} \simeq \mathrm{V}_{4}=\mathrm{C}_{2} \times \mathrm{C}_{2}$}

6.1. Dirichlet series and asymptotic formulas. The results are not difficult. Once again the paper which is cited in this context is [1], which contains several misprints which are corrected in the papers of Mäki [25] and in ours.

Dirichlet series:

$$
\begin{aligned}
\Phi_{4}\left(V_{4}, s\right)= & \frac{1}{6}\left(1+\frac{3}{2^{4 s}}+\frac{6}{2^{6 s}}+\frac{6}{2^{8 s}}\right) \prod_{\substack{p \equiv 1 \\
(\bmod 2)}}\left(1+\frac{3}{p^{2 s}}\right) \\
& -\frac{1}{2} \Phi_{2}\left(C_{2}, 2 s\right)-\frac{1}{6} \\
\Phi_{4,0}\left(V_{4}, s\right)= & \frac{1}{4} \Phi_{4}\left(V_{4}, s\right)-\frac{1}{2} \Phi_{2,0}\left(C_{2}, 2 s\right)+\frac{1}{8} \Phi_{2}\left(C_{2}, 2 s\right)-\frac{1}{8} \\
& +\frac{1}{8}\left(1-\frac{1}{2^{4 s}}+\frac{2}{2^{6 s}}-\frac{2}{2^{8 s}}\right) \prod_{\substack{p \equiv 1 \\
(\bmod 2)}}\left(1+\frac{1+2(-1)^{(p-1) / 2}}{p^{2 s}}\right) \\
\Phi_{2,1}\left(V_{4}, s\right)= & 0 \\
\Phi_{0,2}\left(V_{4}, s\right)= & \Phi_{4}\left(V_{4}, s\right)-\Phi_{4,0}\left(V_{4}, s\right) .
\end{aligned}
$$

Asymptotic formulas:

$$
\begin{aligned}
& N_{4}\left(V_{4}, X\right)=\left(c\left(V_{4}\right) \log ^{2} X+c^{\prime}\left(V_{4}\right) \log X+c^{\prime \prime}\left(V_{4}\right)\right) X^{1 / 2}+R_{4}\left(V_{4}, X\right) \\
& N_{4,0}\left(V_{4}, X\right)=\left(\frac{c\left(V_{4}\right)}{4} \log ^{2} X+\frac{c^{\prime}\left(V_{4}\right)}{4} \log X+\frac{c^{\prime \prime \prime}\left(V_{4}\right)}{4}\right) X^{1 / 2}+R_{4,0}\left(V_{4}, X\right) \\
& N_{2,1}\left(V_{4}, X\right)=0 \\
& N_{0,2}\left(V_{4}, X\right)=\left(\frac{3}{4} c\left(V_{4}\right) \log ^{2} X+\frac{3}{4} c^{\prime}\left(V_{4}\right) \log X+\left(c^{\prime \prime}\left(V_{4}\right)-\frac{c^{\prime \prime \prime}\left(V_{4}\right)}{4}\right)\right) X^{1 / 2} \\
& +R_{0,2}\left(V_{4}, X\right), \quad \text { with } \\
& c\left(V_{4}\right)=\frac{23}{960} \prod_{p}\left(\left(1+\frac{3}{p}\right)\left(1-\frac{1}{p}\right)^{3}\right) \\
& c^{\prime}\left(V_{4}\right)=12 c\left(V_{4}\right)\left(\gamma-\frac{1}{3}+\frac{9 \log 2}{23}+4 \sum_{p \geq 3} \frac{\log p}{(p-1)(p+3)}\right) \\
& c^{\prime \prime}\left(V_{4}\right)=\frac{c^{\prime}\left(V_{4}\right)^{2}}{4 c\left(V_{4}\right)}-\frac{3}{\pi^{2}} \\
& +24 c\left(V_{4}\right)\left(\frac{1}{6}-\gamma_{1}-\frac{\gamma^{2}}{2}-\frac{340}{529} \log ^{2} 2-4 \sum_{p \geq 3} \frac{p(p+1) \log ^{2} p}{(p-1)^{2}(p+3)^{2}}\right)
\end{aligned}
$$




$$
c^{\prime \prime \prime}\left(V_{4}\right)=c^{\prime \prime}\left(V_{4}\right)-\frac{3}{\pi^{2}}+\frac{7}{8 \pi^{2}} \prod_{p \equiv 1} \frac{(1+3 / p)(1-1 / p)}{(1+1 / p)^{2}},
$$

where $\gamma$ is Euler's constant and

$\gamma_{1}=\lim _{n \rightarrow \infty}\left(\sum_{k=1}^{n} \frac{\log k}{k}-\frac{\log ^{2} n}{2 n}\right)=-0.0728158454836767248605863758 \ldots$

Numerically, we have

$$
\begin{aligned}
c\left(V_{4}\right) & =0.0027524302227554813966383118376 \ldots \\
c^{\prime}\left(V_{4}\right) & =0.05137957621042353770883347445 \ldots \\
c^{\prime \prime}\left(V_{4}\right) & =-0.2148583422482281175118362061 \ldots \\
c^{\prime \prime \prime}\left(V_{4}\right) & =-0.4438647800546969108664219885 \ldots
\end{aligned}
$$

Although not difficult to compute, we have not found the additional main terms in the literature.

It is reasonable to conjecture that we should have $R\left(V_{4}, X\right)=\widetilde{O}\left(X^{1 / 4}\right)$, hence to compare with the numerical data we use $\alpha=1 / 4$.

6.2. Tables. These tables have been computed using the methods explained in [8].

\begin{tabular}{|l||l|l|}
\hline$X$ & $N_{4,0}\left(V_{4}, X\right)$ & $N_{0,2}\left(V_{4}, X\right)$ \\
\hline \hline $10^{1}$ & 0 & 0 \\
$10^{2}$ & 0 & 0 \\
$10^{3}$ & 0 & 8 \\
$10^{4}$ & 6 & 41 \\
$10^{5}$ & 42 & 201 \\
$10^{6}$ & 196 & 818 \\
$10^{7}$ & 876 & 3331 \\
$10^{8}$ & 3603 & 13076 \\
$10^{9}$ & 14249 & 50067 \\
$10^{10}$ & 54940 & 187770 \\
$10^{11}$ & 207295 & 694262 \\
$10^{12}$ & 769284 & 2536801 \\
$10^{13}$ & 2814497 & 9167570 \\
$10^{14}$ & 10181802 & 32835581 \\
$10^{15}$ & 36478693 & 116677591 \\
\hline
\end{tabular}




\begin{tabular}{|l||l|l|}
\hline$X$ & $N_{4,0}\left(V_{4}, X\right)$ & $N_{0,2}\left(V_{4}, X\right)$ \\
\hline \hline $10^{16}$ & 129620531 & 411762457 \\
$10^{17}$ & 457321963 & 1444383361 \\
$10^{18}$ & 1603453447 & 5039360330 \\
$10^{19}$ & 5590953378 & 17497040934 \\
$10^{20}$ & 19398735478 & 60486267277 \\
$10^{21}$ & 67009600870 & 208270612830 \\
$10^{22}$ & 230548142363 & 714545063480 \\
$10^{23}$ & 790326082314 & 2443396436299 \\
$10^{24}$ & 2700275901104 & 8329834172525 \\
$10^{25}$ & 9197857451663 & 28317754338743 \\
$10^{26}$ & 31242564815515 & 96017758881843 \\
$10^{27}$ & 105847491463943 & 324784293743259 \\
$10^{28}$ & 357742322840950 & 1096127612328756 \\
$10^{29}$ & 1206393568766650 & 3691598900680670 \\
$10^{30}$ & 4059776186016270 & 12408334995379417 \\
$10^{31}$ & 13635417115241023 & 41630433288940969 \\
$10^{32}$ & 45713153519958996 & 139429524939542248 \\
$10^{33}$ & 152991934395591362 & 466217622608203817 \\
$10^{34}$ & 511204072681788782 & 1556512861826445892 \\
$10^{35}$ & 1705526466144745140 & 5188997592667511054 \\
$10^{36}$ & 5681952310883424255 & 17274863370464181629 \\
\hline
\end{tabular}

The relative error between the actual data and the predictions varies between $-0.73 \%$ and $0.51 \%$, and once again seems clearly to tend to 0 as $k \rightarrow \infty$.

\section{Degree 4 fields with $\mathrm{G} \simeq \mathrm{D}_{4}$}

7.1. Dirichlet series and asymptotic formulas. The results of this section are due to the authors, see [9] and [13]. In the totally complex case (signature $(0,2)$ ) we will distinguish between fields having a real quadratic subfield (using the superscript ${ }^{+}$) and those having a complex quadratic subfield (using the superscript ${ }^{-}$), which gives important extra information (the behavior of the "Frobenius at infinity").

Furthermore, it is convenient both in theory and in practice to introduce the set of imprimitive quartic number fields but now in a fixed algebraic closure of $\mathbb{Q}$, and to denote by $\Phi(I, s)$ and $N(I, X)$ the corresponding $\Phi$ and $N$ functions, possibly with signatures. We then have 


$$
\begin{aligned}
\Phi_{4}(I, s) & =2 \Phi_{4}\left(D_{4}, s\right)+3 \Phi_{4}\left(V_{4}, s\right)+\Phi_{4}\left(C_{4}, s\right) \\
\Phi_{4,0}(I, s) & =2 \Phi_{4,0}\left(D_{4}, s\right)+3 \Phi_{4,0}\left(V_{4}, s\right)+\Phi_{4,0}\left(C_{4}, s\right) \\
\Phi_{2,1}(I, s) & =2 \Phi_{2,1}\left(D_{4}, s\right) \\
\Phi_{0,2}(I, s) & =2 \Phi_{0,2}\left(D_{4}, s\right)+3 \Phi_{0,2}\left(V_{4}, s\right)+\Phi_{0,2}\left(C_{4}, s\right) \\
\Phi_{0,2}^{+}(I, s) & =2 \Phi_{0,2}^{+}\left(D_{4}, s\right)+\Phi_{0,2}\left(V_{4}, s\right)+\Phi_{0,2}\left(C_{4}, s\right) \\
\Phi_{0,2}^{-}(I, s) & =2 \Phi_{0,2}^{-}\left(D_{4}, s\right)+2 \Phi_{0,2}\left(V_{4}, s\right),
\end{aligned}
$$

and the same linear combinations for the $N$ functions. The last two formulas come from the fact that the quadratic subfield of a $C_{4}$ field is always real and that a complex $V_{4}$ field always contains two complex and one real quadratic subfield.

Thus we will give the formulas only for $I$ (the above combinations allowing to easily get back to $D_{4}$ ), but the tables only for $D_{4}$. Note the important fact that, as a consequence, the asymptotic constants for $D_{4}$ are one half of the ones for $I$.

Denote by $\mathcal{D}$ the set of all fundamental discriminants, in other words 1 and discriminants of quadratic fields. For any $d \in \mathcal{D}$, denote by $L(s, d)$ the Dirichlet $L$-series for the quadratic character $\left(\frac{d}{n}\right)$.

Dirichlet series:

$$
\Phi_{4}(I, s)=\frac{1}{2 \zeta(2 s)} \sum_{D \in \mathcal{D} \backslash\{1\}} \frac{2^{-r_{2}(D)}}{|D|^{2 s} L(2 s, D)} F_{D}(s)-\Phi_{2}\left(C_{2}, 2 s\right),
$$

where $r_{2}(D)=0$ if $D>0, r_{2}(D)=1$ if $D<0$, with

$$
\begin{aligned}
F_{D}(s)= & \sum_{\substack{d \mid D, d \in \mathcal{D}, \operatorname{gcd}(d, D / d)=1 \\
d>0 \text { if } D>0}} f_{D, d}(s) L(s, d) L(s, D / d) \\
& +g_{D}(s) \sum_{\substack{d \mid D, d \in \mathcal{D}, \operatorname{gcd}(d, D / d)=1 \\
k(D) d>0 \text { if } D>0}} L(s, k(D) d) L(s, k(D) D / d),
\end{aligned}
$$

where

$$
f_{D, d}(s)= \begin{cases}1-\frac{1}{2^{2 s}}+\frac{4}{2^{4 s}} & \text { if } D \equiv 5(\bmod 8) \\ 1-\frac{2\left(\frac{d}{2}\right)}{2^{s}}+\frac{5}{2^{2 s}}-\frac{4\left(\frac{d}{2}\right)}{2^{3 s}}+\frac{4}{2^{4 s}} & \text { if } D \equiv 1(\bmod 8) \\ 1-\frac{\left(\frac{d_{1}}{2}\right)}{2^{s}}+\frac{2}{2^{2 s}}-\frac{2\left(\frac{d_{1}}{2}\right)}{2^{3 s}}+\frac{4}{2^{4 s}} & \text { if } D \equiv 0(\bmod 4),\end{cases}
$$


where $d_{1}=d$ if $d \equiv 1(\bmod 4), d_{1}=D / d$ if $d \equiv 0(\bmod 4)$,

$$
\begin{gathered}
k(D)= \begin{cases}-4 & \text { if } D \not \equiv-4(\bmod 16) \\
8 & \text { if } D \equiv-4(\bmod 16),\end{cases} \\
g_{D}(s)= \begin{cases}1 & \text { if } D \not \equiv 8(\bmod 16) \\
1+\frac{2}{2^{2 s}} & \text { if } D \equiv 8(\bmod 16) .\end{cases}
\end{gathered}
$$

The Dirichlet series for $\Phi_{r_{1}, r_{2}}(I, s)$ are of a similar nature but are too complicated to be given here (see [9]).

Asymptotic formulas:

$$
\begin{aligned}
N_{4}(I, X) & =2 c\left(D_{4}\right) X+R_{4}\left(D_{4}, X\right) \\
N_{4,0}(I, X) & =\frac{c^{+}\left(D_{4}\right)}{2} X+R_{4,0}(I, X) \\
N_{2,1}(I, X) & =c^{+}\left(D_{4}\right) X+R_{2,1}(I, X) \\
N_{0,2}(I, X) & =\frac{c^{+}\left(D_{4}\right)+2 c^{-}\left(D_{4}\right)}{2} X+R_{0,2}(I, X) \\
N_{0,2}^{+}(I, X) & =\frac{c^{+}\left(D_{4}\right)}{2} X+R_{0,2}^{+}(I, X) \\
N_{0,2}^{-}(I, X) & =c^{-}\left(D_{4}\right) X+R_{0,2}^{-}(I, X),
\end{aligned}
$$

with

$$
\begin{aligned}
c^{ \pm}\left(D_{4}\right) & =\frac{3}{\pi^{2}} \sum_{\operatorname{sign}(D)= \pm} \frac{1}{D^{2}} \frac{L(1, D)}{L(2, D)} \text { and } \\
c\left(D_{4}\right) & =c^{+}\left(D_{4}\right)+\frac{c^{-}\left(D_{4}\right)}{2},
\end{aligned}
$$

where the sum is over discriminants $D$ of quadratic fields of given sign.

Numerically, we have

$$
\begin{aligned}
c^{+}\left(D_{4}\right) & =0.01971137577, \quad c^{-}\left(D_{4}\right)=0.06522927087, \\
c\left(D_{4}\right) & =0.05232601119,
\end{aligned}
$$

where in each case the mean deviation seems to be less than 100 in the last given digit (i.e., $\pm 10^{-9}$ ).

It is possible that these constants can be expressed as finite linear combinations of simple Euler products, but we have not been able to find such expressions.

It can be shown (see [13]) that $R(I, X)=\widetilde{O}\left(X^{3 / 4}\right)$, and it is reasonable to conjecture that we should have $R(I, X)=\widetilde{O}\left(X^{1 / 2}\right)$. However, the tables 
seem to show that there are additional main terms, so that

$$
R(I, X)=2\left(c^{\prime}\left(D_{4}\right) \log X+c^{\prime \prime}\left(D_{4}\right)\right) X^{1 / 2}+O\left(X^{\alpha}\right)
$$

for suitable constants $c_{R_{1}, R_{2}}^{\prime}\left(D_{4}\right)$ and $c_{R_{1}, R_{2}}^{\prime \prime}\left(D_{4}\right)$ (depending on the signature), and some $\alpha<1 / 2$ (we include an extra factor 2 above so that it disappears in the formulas for $D_{4}$ ).

A least squares computation gives

$$
\begin{aligned}
c^{\prime}\left(D_{4}\right) & =0.034067 & c^{\prime \prime}\left(D_{4}\right) & =-0.81992 \\
c_{4,0}^{\prime}\left(D_{4}\right) & =0.0092312 & c_{4,0}^{\prime \prime}\left(D_{4}\right) & =-0.26410 \\
c_{2,1}^{\prime}\left(D_{4}\right) & =-0.0030683 & c_{2,1}^{\prime \prime}\left(D_{4}\right) & =-0.027401 \\
c_{0,2}^{\prime}\left(D_{4}\right) & =0.027904 & c_{0,2}^{\prime \prime}\left(D_{4}\right) & =-0.52842 \\
c_{0,2}^{\prime+}\left(D_{4}\right) & =0.0096442 & c^{\prime \prime+}{ }_{0,2}\left(D_{4}\right) & =-0.13795 \\
c_{0,2}^{\prime-}\left(D_{4}\right) & =0.018260 & c^{\prime \prime}{ }_{0,2}^{-}\left(D_{4}\right) & =-0.39047
\end{aligned}
$$

Here, even though we give the values with 5 digits, they are probably accurate only to within a factor of 2 or so. Nevertheless, the least square fit is very good, hence we use these values to compare with the actual data.

This seems to show that the functions $\Phi(I, s)$ have a double pole at $s=1 / 2$, but we do not know how to prove this or how to compute the polar parts at $s=1 / 2$, although heuristically it is easy to guess why they have at least a simple pole.

Thus, to compare with the data we use these refined estimates, and we choose $\alpha=2 / 5$, which seems to give reasonable results.

7.2. Tables. See [13] and [9] for the methods used to compute these tables.

\begin{tabular}{|l||l|l|l|}
\hline$X$ & $N_{4,0}\left(D_{4}, X\right)$ & $N_{2,1}\left(D_{4}, X\right)$ & $N_{0,2}\left(D_{4}, X\right)$ \\
\hline \hline $10^{1}$ & 0 & 0 & 0 \\
$10^{2}$ & 0 & 0 & 0 \\
$10^{3}$ & 1 & 6 & 17 \\
$10^{4}$ & 25 & 93 & 295 \\
$10^{5}$ & 379 & 968 & 3417 \\
$10^{6}$ & 4486 & 9772 & 36238 \\
$10^{7}$ & 47562 & 98413 & 370424 \\
$10^{8}$ & 486314 & 984708 & 3734826 \\
$10^{9}$ & 4903607 & 9852244 & 37469573 \\
$10^{10}$ & 49188349 & 98546786 & 375154025 \\
\hline
\end{tabular}




\begin{tabular}{|l||l|l|l|}
\hline$X$ & $N_{4,0}\left(D_{4}, X\right)$ & $N_{2,1}\left(D_{4}, X\right)$ & $N_{0,2}\left(D_{4}, X\right)$ \\
\hline \hline $10^{11}$ & 492454432 & 985536549 & 3753258277 \\
$10^{12}$ & 4926654580 & 9855572218 & 37538880690 \\
$10^{13}$ & 49274156836 & 98556488881 & 375411901218 \\
$10^{14}$ & 492769145545 & 985567509497 & 3754202033198 \\
$10^{15}$ & 4927790007755 & 9855683662056 & 37542317217650 \\
$10^{16}$ & 49278249627160 & 98556864596086 & 375424223055946 \\
$10^{17}$ & 492783730187748 & 985568739794773 & 3754245940051259 \\
\hline
\end{tabular}

\begin{tabular}{|l||l|l|}
\hline$X$ & $N_{0,2}^{+}\left(D_{4}, X\right)$ & $N_{0,2}^{-}\left(D_{4}, X\right)$ \\
\hline \hline $10^{1}$ & 0 & 0 \\
$10^{2}$ & 0 & 0 \\
$10^{3}$ & 0 & 17 \\
$10^{4}$ & 27 & 268 \\
$10^{5}$ & 395 & 3022 \\
$10^{6}$ & 4512 & 31726 \\
$10^{7}$ & 47708 & 322716 \\
$10^{8}$ & 486531 & 3248295 \\
$10^{9}$ & 4904276 & 32565297 \\
$10^{10}$ & 49190647 & 325963378 \\
$10^{11}$ & 492464630 & 3260793647 \\
$10^{12}$ & 4926673909 & 32612206781 \\
$10^{13}$ & 49274235813 & 326137665405 \\
$10^{14}$ & 492769387400 & 3261432645798 \\
$10^{15}$ & 4927790822970 & 32614526394680 \\
$10^{16}$ & 49278252225484 & 326145970830462 \\
$10^{17}$ & 492783738112277 & 3261462201938982 \\
\hline
\end{tabular}

The relative error between the actual data and the predictions varies between $-0.32 \%$ and $0.76 \%$.

\section{Degree 4 fields with $\mathrm{G} \simeq \mathrm{A}_{4}$}

8.1. Dirichlet series and asymptotic formulas. Using Kummer theory, it is possible to obtain an explicit expression for the Dirichlet series $\Phi_{4}\left(A_{4}, k, s\right)$ where the additional parameter $k$ indicates that we fix the resolvent cubic field (see [11]), hence an asymptotic formula for $N_{4}\left(A_{4}, k, s\right)$. However, as indicated in loc. cit., it does not seem possible to sum naively on $k$ to obtain an asymptotic estimate for $N_{4}\left(A_{4}, X\right)$. Thus we must be content with experimental data. According to general conjectures, including that of Malle, it is reasonable to conjecture that we have an asymptotic 
formula of the form

$$
N_{4}\left(A_{4}, X\right) \sim c\left(A_{4}\right) X^{1 / 2} \log X
$$

for some constant $c\left(A_{4}\right)>0$. As for the case $G \simeq D_{4}$, it is possible that the constant $c\left(A_{4}\right)$ can be expressed as a finite linear combination of Euler products with explicit coefficients. In view of the numerical data, it is possible that we have a sharper formula of the form

$$
N_{4}\left(A_{4}, X\right)=\left(c\left(A_{4}\right) \log X+c^{\prime}\left(A_{4}\right)\right) X^{1 / 2}+O\left(X^{\alpha}\right)
$$

for some $\alpha<1 / 2$, perhaps for any $\alpha>1 / 4$. We obtain an excellent least squares fit by using $c\left(A_{4}\right)=0.018634$ and $c^{\prime}\left(A_{4}\right)=-0.14049$. We obtain similar fits for the tables with signatures $\left(c_{4,0}\left(A_{4}\right)=0.0049903\right.$, $c_{4,0}^{\prime}\left(A_{4}\right)=-0.0373357, c_{0,2}\left(A_{4}\right)=0.0136441, c_{0,2}^{\prime}\left(A_{4}\right)=-0.103157$ with evident notations). All these values should be correct to within $5 \%$.

To compare with the numerical data we use the values obtained above with the least squares fit and we choose $\alpha=1 / 4$, which gives reasonable results.

8.2. Numerical computation. We have generated $A_{4}$ extensions using Kummer theory of quadratic extensions over cyclic cubic fields, keeping only those extensions whose discriminant is less than the required bound (see [14] for details). The computations without signatures being simpler than with signatures have been pushed to $X=10^{16}$, while those with signatures have only been pushed to $X=10^{13}$, although it should be easy to push them further. Thus, exceptionally we also give separately the data without signature distinction.

8.3. Tables. See [14] for the methods used to compute these tables.

\begin{tabular}{|l||l||l||l|}
\hline$X$ & $N_{4}\left(A_{4}, X\right)$ & $X$ & $N_{4}\left(A_{4}, X\right)$ \\
\hline \hline $10^{1}$ & 0 & $10^{9}$ & 7699 \\
$10^{2}$ & 0 & $10^{10}$ & 28759 \\
$10^{3}$ & 0 & $10^{11}$ & 104766 \\
$10^{4}$ & 4 & $10^{12}$ & 374470 \\
$10^{5}$ & 27 & $10^{13}$ & 1319606 \\
$10^{6}$ & 121 & $10^{14}$ & 4602909 \\
$10^{7}$ & 514 & $10^{15}$ & 15915694 \\
$10^{8}$ & 2010 & $10^{16}$ & 54592313 \\
\hline
\end{tabular}




\begin{tabular}{|l||l|l|}
\hline$X$ & $N_{4,0}\left(A_{4}, X\right)$ & $N_{0,2}\left(A_{4}, X\right)$ \\
\hline \hline $10^{1}$ & 0 & 0 \\
$10^{2}$ & 0 & 0 \\
$10^{3}$ & 0 & 0 \\
$10^{4}$ & 0 & 4 \\
$10^{5}$ & 4 & 23 \\
$10^{6}$ & 31 & 90 \\
$10^{7}$ & 129 & 385 \\
$10^{8}$ & 527 & 1483 \\
$10^{9}$ & 2037 & 5662 \\
$10^{10}$ & 7662 & 21097 \\
$10^{11}$ & 28182 & 76584 \\
$10^{12}$ & 100576 & 273894 \\
$10^{13}$ & 354302 & 965304 \\
\hline
\end{tabular}

The relative error between the actual data and the predictions varies between $-1.01 \%$ and $1.03 \%$.

\section{Degree 4 fields with $\mathrm{G} \simeq \mathrm{S}_{4}$}

9.1. Dirichlet series and asymptotic formulas. By using similar methods to the $A_{4}$ case but this time with Kummer theory over noncyclic cubic fields, we can also compute explicitly the Dirichlet series $\Phi_{4}\left(S_{4}, k, s\right)$, which is quite similar in form to $\Phi_{4}\left(A_{4}, k, s\right)$, where $k$ is a fixed cubic resolvent, see once again [11], hence also obtain an asymptotic formula for $N_{4}\left(S_{4}, k, X\right)$, with evident notation. Contrary to the $A_{4}$ case, however, it seems that it is now possible to sum the contributions coming from the different cubic resolvents and obtain an asymptotic formula for $N_{4}\left(S_{4}, X\right)$. However this does not give a very useful formula, neither in theory nor for numerical computation, and in any case is completely superseded by the work of Bhargava.

Indeed, in a series of groundbreaking papers [5], Bhargava gives a wide generalization of the methods of Davenport-Heilbronn and as a consequence obtains an asymptotic formula for $N\left(S_{4}, X\right)$, including a simple expression for $c\left(S_{4}\right)$, and also with signatures.

Asymptotic formulas:

$$
\begin{aligned}
N_{4}\left(S_{4}, X\right)= & r_{4}\left(S_{4}\right) z\left(S_{4}\right) X+c^{\prime}\left(S_{4}\right) X^{5 / 6} \\
& +\left(c^{\prime \prime}\left(S_{4}\right) \log X+c^{\prime \prime \prime}\left(S_{4}\right)\right) X^{3 / 4}+R_{4}\left(S_{4}, X\right) \\
N_{r_{1}, r_{2}}\left(S_{4}, X\right)= & r_{r_{1}, r_{2}}\left(S_{4}\right) z\left(S_{4}\right)+c^{\prime}{ }_{r_{1}, r_{2}}\left(S_{4}\right) X^{5 / 6} \\
& +\left(c^{\prime \prime}{ }_{r_{1}, r_{2}}\left(S_{4}\right) \log X+c^{\prime \prime \prime}{ }_{r_{1}, r_{2}}\left(S_{4}\right)\right) X^{3 / 4}+R_{r_{1}, r_{2}}\left(S_{4}, X\right)
\end{aligned}
$$


with

$z\left(S_{4}\right)=\prod_{p \geq 2}\left(1+\frac{1}{p^{2}}-\frac{1}{p^{3}}-\frac{1}{p^{4}}\right)=1.2166902869063309337694390868 \ldots$

and

$$
r_{4}\left(S_{4}\right)=\frac{5}{24}, r_{4,0}\left(S_{4}\right)=\frac{1}{48}, r_{2,1}\left(S_{4}\right)=\frac{1}{8}, r_{0,2}\left(S_{4}\right)=\frac{1}{16} .
$$

The additional main terms given above have been suggested to the authors in a personal communication of Yukie.

Bhargava proves only that $R\left(S_{4}, X\right)=o(X)$, but conjecturally we should have $R\left(S_{4}, X\right)=O\left(X^{\alpha}\right)$ for some $\alpha<3 / 4$, perhaps for any $\alpha>1 / 2$. We will use $\alpha=1 / 2$ for comparisons with the actual data.

As in the $D_{4}$ and $A_{4}$ cases, we can try using a least squares method to find the additional main terms. However, note first that, using our Kummertheoretic method, it is very costly to compute $N\left(S_{4}, 10^{7}\right)$ since it involves in particular computing the class group and units of 2.5 million (exactly $N_{3}\left(S_{3}, 10^{7}\right)$ ) cubic fields (see below, however). Second, note that in the range $X \leq 10^{7}$ the functions $X, X^{5 / 6}, X^{3 / 4} \log X$, and $X^{3 / 4}$ are quite close to one another (for instance the function $X^{3 / 4} \log X$ which is asymptotically negligible with respect to $X^{5 / 6}$ is still more than 4 times larger for $X=10^{7}$ ), hence it will be almost impossible to distinguish between their coefficients using a least squares method. Nonetheless we have done so and found

$$
\begin{aligned}
& c^{\prime}\left(S_{4}\right)=-2.17561, \quad c^{\prime \prime}\left(S_{4}\right)=0.08417, \quad c^{\prime \prime \prime}\left(S_{4}\right)=1.91916 \\
& c_{4,0}^{\prime}\left(S_{4}\right)=-0.42792, \quad c^{\prime \prime}{ }_{4,0}\left(S_{4}\right)=0.034743, \quad c^{\prime \prime}{ }_{4,0}\left(S_{4}\right)=0.33335 \\
& c^{\prime}{ }_{2,1}\left(S_{4}\right)=-1.28495, \quad c^{\prime \prime}{ }_{2,1}\left(S_{4}\right)=0.051021, \quad c^{\prime \prime}{ }_{2,1}\left(S_{4}\right)=1.11068 \\
& c_{0,2}^{\prime}\left(S_{4}\right)=-0.46274, \quad c^{\prime \prime}{ }_{0,2}\left(S_{4}\right)=-0.001590, \quad c^{\prime \prime}{ }_{0,2}\left(S_{4}\right)=0.47514 \text {. }
\end{aligned}
$$

These values are only given to indicate how the tables have been computed, but are certainly very far from the correct ones.

9.2. Numerical computation. As for $A_{4}$ extensions, we use Kummer theory of quadratic extensions, this time over noncyclic cubic fields and we keep only those extensions whose discriminant is less than the required bound. See [14] for details. The reason that we cannot easily go above $10^{7}$ is that we need to compute units and class groups for all (noncyclic) cubic fields of discriminant up to that bound, and this is very time-consuming. It is possible that in the same way that Belabas adapted the methods of Davenport-Heilbronn to compute rapidly tables of $S_{3}$-cubic fields by enumerating reduced cubic forms, one can adapt the method of Bhargava to efficiently compute $S_{4}$-quartic fields by enumerating reduced pairs of ternary quadratic forms, thus enabling computations to much larger discriminant bounds. 
9.3. Tables. It should be emphasized that contrary to the other Galois groups, our numerical predictions should here be only considered as guesses.

\begin{tabular}{|l||l|l|l|}
\hline$X$ & $N_{4,0}\left(S_{4}, X\right)$ & $N_{2,1}\left(S_{4}, X\right)$ & $N_{0,2}\left(S_{4}, X\right)$ \\
\hline \hline $10^{1}$ & 0 & 0 & 0 \\
$10^{2}$ & 0 & 0 & 0 \\
$10^{3}$ & 0 & 10 & 8 \\
$10^{4}$ & 13 & 351 & 206 \\
$10^{5}$ & 449 & 5916 & 3374 \\
$10^{6}$ & 8301 & 80899 & 44122 \\
$10^{7}$ & 120622 & 989587 & 525099 \\
\hline
\end{tabular}

The relative error between the actual data and the predictions varies between $-0.18 \%$ and $0.28 \%$.

For instance, our estimates give $P_{4}\left(S_{4}, 10^{8}\right)=18719128, P_{4,0}\left(S_{4}, 10^{8}\right)=$ 1521877, $P_{2,1}\left(S_{4}, 10^{8}\right)=11294945, P_{0,2}\left(S_{4}, 10^{8}\right)=5902307$. It would be interesting to see how close to the truth are these estimates.

Note added: we have just learnt that in a large computation using Hunter's method instead of Kummer theory, G. Malle has computed

$$
N_{4,0}\left(S_{4}, 10^{8}\right)=1529634 \text { and } N_{4,0}\left(S_{4}, 10^{9}\right)=17895702,
$$

so our above prediction for $10^{8}$ was within $0.5 \%$ of the correct value.

\section{References}

[1] A. BAILY, On the density of discriminants of quartic fields. J. reine angew. Math. 315 (1980), 190-210.

[2] K. Belabas, A fast algorithm to compute cubic fields. Math. Comp. 66 (1997), 1213-1237.

[3] K. Belabas, On quadratic fields with large 3-rank. Math. Comp. 73 (2004), 2061-2074.

[4] K. Belabas, M. Bhargava, C. Pomerance, Error estimates for the Davenport-Heilbronn theorems. Preprint available at http://www.math.u-bordeaux1.fr/ belabas/pub/\#BPP

[5] M. Bhargava, Higher Composition Laws I, II, III, IV.

[6] H. Cohen, A course in computational algebraic number theory (fourth printing). GTM 138, Springer-Verlag, 2000.

[7] H. CoHen, Advanced topics in computational number theory. GTM 193, Springer-Verlag, 2000.

[8] H. Cohen, Comptage exact de discriminants d'extensions abéliennes. J. Th. Nombres Bordeaux 12 (2000), 379-397.

[9] H. Cohen, Enumerating quartic dihedral extensions of $\mathbb{Q}$ with signatures. Ann. Institut Fourier 53 (2003) 339-377.

[10] H. CoHen, High precision computation of Hardy-Littlewood constants. Preprint available on the author's web page at the URL http://www.math.u-bordeaux.fr/ cohen.

[11] H. Cohen, Counting $\mathrm{A}_{4}$ and $\mathrm{S}_{4}$ extensions of number fields with given resolvent cubic, in "High primes and misdemeanours: lectures in honour of the 60th birthday of Hugh Cowie Williams". Fields Institute Comm. 41 (2004), 159-168.

[12] H. Cohen, Constructing and counting number fields. Proceedings ICM 2002 Beijing vol II, Higher Education Press, China (2002), 129-138. 
[13] H. Cohen, F. Diaz y Diaz, M. Olivier, Enumerating quartic dihedral extensions of $\mathbb{Q}$. Compositio Math. 133 (2002), 65-93.

[14] H. Cohen, F. Diaz y Diaz, M. Olivier, Construction of tables of quartic fields using Kummer theory. Proceedings ANTS IV, Leiden (2000), Lecture Notes in Computer Science 1838, Springer-Verlag, 257-268.

[15] H. Cohen, F. Diaz y DiAz, M. Olivier, Constructing complete tables of quartic fields using Kummer theory. Math. Comp. 72 (2003) 941-951.

[16] H. Cohen, F. Diaz y Diaz, M. Olivier, Densité des discriminants des extensions cycliques de degré premier. C. R. Acad. Sci. Paris 330 (2000), 61-66.

[17] H. Cohen, F. Diaz y Diaz, M. Olivier, On the density of discriminants of cyclic extensions of prime degree. J. reine angew. Math. 550 (2002), 169-209.

[18] H. Cohen, F. Diaz y Diaz, M. Olivier, Cyclotomic extensions of number fields. Indag. Math. (N.S.) 14 (2003), 183-196.

[19] H. Cohn, The density of abelian cubic fields. Proc. Amer. Math. Soc. 5 (1954), 476-477.

[20] B. Datskovsky, D. J. WRight, Density of discriminants of cubic extensions. J. reine angew. Math. 386 (1988), 116-138.

[21] H. Davenport, H. Heilbronn, On the density of discriminants of cubic fields I. Bull. London Math. Soc. 1 (1969), 345-348.

[22] H. Davenport, H. Heilbronn, On the density of discriminants of cubic fields II. Proc. Royal. Soc. A 322 (1971), 405-420.

[23] H. Hasse, Arithmetische Theorie der kubischen Zahlkörper auf klassenkörpertheoretischer Grundlage. Math. Zeitschrift 31 (1930), 565-582.

[24] J. KLÜNERS, A counter-example to Malle's conjecture on the asymptotics of discriminants. C. R. Acad. Sci. Paris 340 (2005), 411-414.

[25] S. MäKI, On the density of abelian number fields. Thesis, Helsinki, 1985.

[26] S. M̈̈KI, The conductor density of abelian number fields. J. London Math. Soc. (2) 47 (1993), 18-30.

[27] G. MALle, On the distribution of Galois groups. J. Number Th. 92 (2002), 315-329.

[28] G. Malle, On the distribution of Galois groups II, Exp. Math. 13 (2004), 129-135.

[29] G. MALle, The totally real primitive number fields of discriminant at most $10^{9}$. Proceedings ANTS VII (Berlin), 2006, Springer Lecture Notes in Computer Science XXX.

[30] D. Roberts, Density of cubic field discriminants. Math. Comp. 70 (2001), 1699-1705.

[31] G. Tenenbaum, Introduction à la théorie analytique et probabiliste des nombres. Cours Spécialisés SMF 1, Société Mathématique de France, 1995.

[32] D. J. Wright, Distribution of discriminants of Abelian extensions. Proc. London Math. Soc. (3) 58 (1989), 17-50.

[33] D. J. WRIGHT, personal communication.

[34] D. J. WRIGHT, A. YUKIE, Prehomogeneous vector spaces and field extensions. Invent. Math. 110 (1992), 283-314.

Henri Cohen, Francisco Diaz y Diaz, Michel Olivier

Laboratoire A2X, U.M.R. 5465 du C.N.R.S.,

Université Bordeaux I, 351 Cours de la Libération,

33405 TALENCE Cedex, FRANCE

E-mail : cohen,diaz,olivier@math.u-bordeaux1.fr 